\title{
EVALUATION OF PATIENT EXPOSURE AFTER AN EMERGENCY WARM-UP OF THE CT X-RAY TUBE
}

\author{
Desislava Z. Kostova- \\ Lefterova ${ }^{1,2}$, \\ Desislava P. Ivanova ${ }^{3,4}$, \\ Filip N. Simeonov 5 , \\ Anna R. Zagorska ${ }^{6}$, \\ Kameliya Z. Genova, \\ Petya K. Ilieva
}

\author{
National Cardiology Hospital - Sofia \\ ${ }^{1}$ University Hospital Aleksandrovska - \\ Sofia \\ ${ }^{2}$ Medical University-Pleven \\ ${ }^{3}$ St. Ekaterina University Hospital - \\ Sofia \\ ${ }^{4}$ Military Hospital - Sofia \\ ${ }^{5}$ National Centre of Radiobiology and \\ Radiation Protection - Sofia \\ ${ }^{6}$ Acibadem City Clinic Tokuda Hospital \\ - Sofia
}

\author{
Corresponding Author: \\ Desislava Z. Kostova-Lefterova \\ Medical Imaging Department, \\ National Cardiology Hospital \\ 65, Konyovitsa Str. \\ Sofia, 1309 \\ Bulgaria \\ e-mail:dessi.zvkl@gmail.com
}

Received: June 19, 2020

Revision received: September 02, 2020

Accepted: December 11, 2020

\section{Summary}

A survey was performed to evaluate patient exposure after a procedure for the CT X-ray tube's emergency warm-up, with the patient positioned on the table within the CT gantry. Three CT units, situated in three different University hospitals, were included in the survey. The evaluation was performed with AGFA personal monitoring films to visualize the results and discuss them with the radiographers who operate the systems. Additional measurements were performed with the RaySafe X2 system to demonstrate the presence of exposure. The air kerma resulting from implementing the warm-up protocol was evaluated to be higher than $112 \mu \mathrm{Gy}, 409 \mu \mathrm{Gy}$, and 807 $\mu \mathrm{Gy}$ for each of the CT units. Those values were underestimated because of the dosimetry equipment used and the methodology. A discussion between medical staff, engineers, and medical physicist was initiated. The practice with unnecessary patient exposure was terminated.

Keywords: patient dosimetry, computed tomography, X-ray tube, warming procedure, patient exposure

\section{Introduction}

Computed Tomography (CT) has been technically significantly improved as a medical imaging modality since its introduction into clinical practice. The engineering improvements have led to many important clinical applications and a massive impact in patient care [1]. However, this progress has come at a price: exposing patients to potentially dangerous ionizing radiation [2], with the increased CT radiation exposure been of growing concern in recent years [3]. The operation of a CT unit also requires 1) radiologists' and technologists' detailed and profound knowledge of the CT scanners' potential in order to fully exploit it and to avoid patient overexposure at the same time; 2) service engineers that can interpret the wide variety of the technical instructions and work with different variables while ensuring the appropriate communication with the customer 
Table 1. Description of CT units

\begin{tabular}{cccc}
\hline Hospital & Unit & Year of installation & Designation \\
\hline $\mathbf{1}$ & 64 slices & 2008 & $\mathrm{~A}$ \\
\hline $\mathbf{2}$ & 64 slices & 2009 & $\mathrm{~B}$ \\
\hline $\mathbf{3}$ & 320 slices & 2009 & $\mathrm{C}$ \\
\hline
\end{tabular}

and provide service and operation within the applicable regulatory requirements; 3 ) medical physicists as members of the medical team improve the radiation protection and help to prevent wrong radiation protection practices.

The purpose of this study was: 1) to present and analyze the practice to perform an emergency warm-up of the CT scanner with a patient within the CT gantry, that was observed in three different hospitals; 2) to present a simple way of communication with the medical staff in order to prevent such practices.

\section{Warm-up procedure}

The purpose of an X-ray tube's warm-up procedure is to avoid damage to the X-ray tube and avoid any artifacts in the images acquired. A CT is a high-powered machine, and the anode's cooling is achieved by the circulation of oil through it to a heat exchanger. If a cold anode is exposed to high-powered energy, anode damage often occurs. A general recommendation is proposed by all manufacturers for warming of the X-ray tubes by different series of low energy exposures to prevent such type of damage [4]. The $\mathrm{X}$-ray tube's heating procedure is performed by carefully increasing the tube current and voltage, leading to the slow burning of any oxygen molecules present before reaching a safe level for high-powered operation of the tube. The tube is warmed up gradually in a controlled manner, so the tube can reach its operating temperature before scanning a patient. The CT manufacturers have recommended in the respective $\mathrm{CT}$ manuals never to perform scanner calibration, scanner testing, or tube heating when a patient or other personnel are present in the CT room.

\section{Materials and Methods}

The investigation was performed in three University hospitals in Bulgaria (Table 1), where medical physicists are employed. The study was performed in two different stages. The first stage was a qualitative assessment of patient exposure during CT warm-up. The second stage was to measure the air kerma and air kerma rate during the warm-up procedure and to compare the results between all three scanners. All CT units are from the same manufacturer with the same practice for warming the X-ray tube with a patient inside the gantry. A short description of the CT systems is presented in Table 1 .

\section{Qualitative assessment}

For qualitative detection, educational purposes for the radiographers, and visual demonstration, AGFA Personal Monitoring films (Agfa-Gevaert Group, Mortsel, Belgium) were used during the warm-up protocols for two of the investigated CT units (A and C). Each film contained two layers of slow and fast emulsion, which allowed the

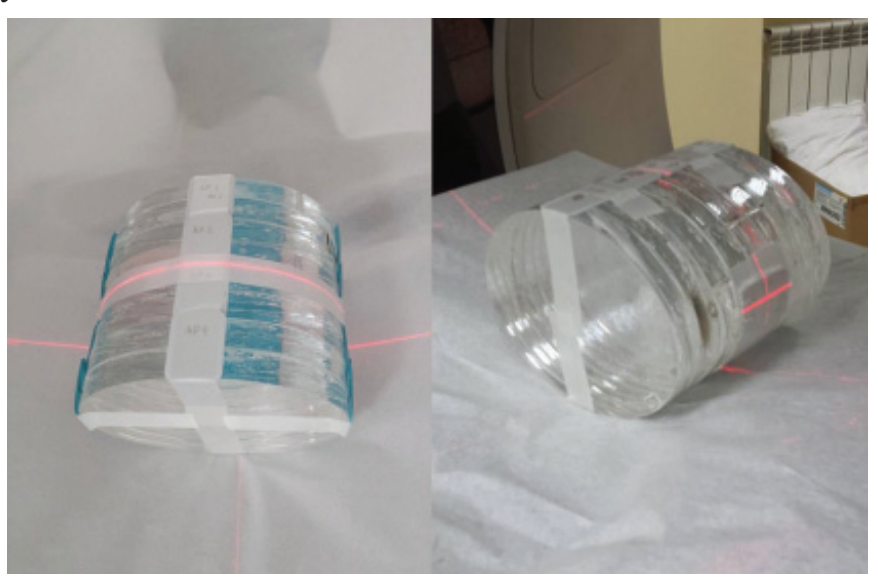

Figure 1. Positioning of the films along the ellipsoid PMMA phantom a) CT Unit A, b) CT unit C 
detection of the ionizing radiation over a wide air kerma range [5]. The films were stacked one by one in groups of four with overlapping of about $2 \mathrm{~mm}$. The film groups were positioned at four sides of the phantom - the anterior, the posterior, the lateral-sinistral, and the lateral-dextral, all of them aligned along the $\mathrm{z}$-direction at the surface of the ellipsoid homemade PMMA phantom and exposed during the warm-up process (Fig. 1). The phantom represents a small adult patient.

The experimental set up during the first phase of the study is presented in Figure 1.

\section{Measurements}

Measurements were performed with RaySafe X2 (Unfors RaySafe AB, Sweden). The active dosimeter used for the survey was the X2 R/F sensor, calibrated by the manufacturer in SSDL. The sensor is calibrated to measure radiological parameters such as dose, dose rate, tube voltage, half-value layer, total filtration, exposure time, pulses, pulse rate, and dose/pulse - in a single exposure for radiography. The $\mathrm{X} 2 \mathrm{R} / \mathrm{F}$ sensor can also be used for $\mathrm{kVp}$ and HVL measurements on CT machines. It is not an exact tool for dose measurements on $\mathrm{CT}$, but the purpose of the survey was to demonstrate that there is exposure during the warm-up procedure with the departments' equipment.

\section{Results}

A routine practice for warming the CT X-ray tube with the patient positioned on the table within the gantry was performed in the surveyed CT units. The reason for this practice was that the radiographers were taught that the warming process should always be performed when the collimator was closed collimator to guarantee that the patient is never exposed to radiation. A measurement of the collimator shielding was never performed by a qualified service engineer or medical physicist. A discussion between medical physicists, technicians, and engineers was initiated, but practices were not changed. A qualitative assessment of patient exposure was performed to prove that a missing image on the CT monitor display does not mean the patient is not exposed to irradiation.

\section{Qualitative assessment}

Two units were included in this part of the study: CT unit A and CT unit $\mathrm{C}$, where such a warming practice by the radiographers was observed multiple times.

The results from the experimental set up (Figure 1), are presented in Figure 2.

The qualitative assessment presented in Fig. 2 demonstrates the presence of exposure during the warm-up procedure. Although the units are from the same manufacturer, the warmup protocols are different and cover at least 16 $\mathrm{cm}$ of irradiated field. The results show that the x-ray tube is stationary for CT unit $\mathrm{C}$ with a larger irradiated field than unit $\mathrm{A}$ and that it rotates continuously for $\mathrm{CT}$ in unit $\mathrm{A}$ but with a smaller slice thickness.

\section{Measurements with RaySafe X2}

The second part of the study was to evaluate the air kerma during the warm-up procedure. The air kerma measurements with RaySafe X2 are presented in Figure 3 and Figure 4.

The values for the total measured air kerma value and exposure time for completed warming procedure per CT unit were respectively: 112

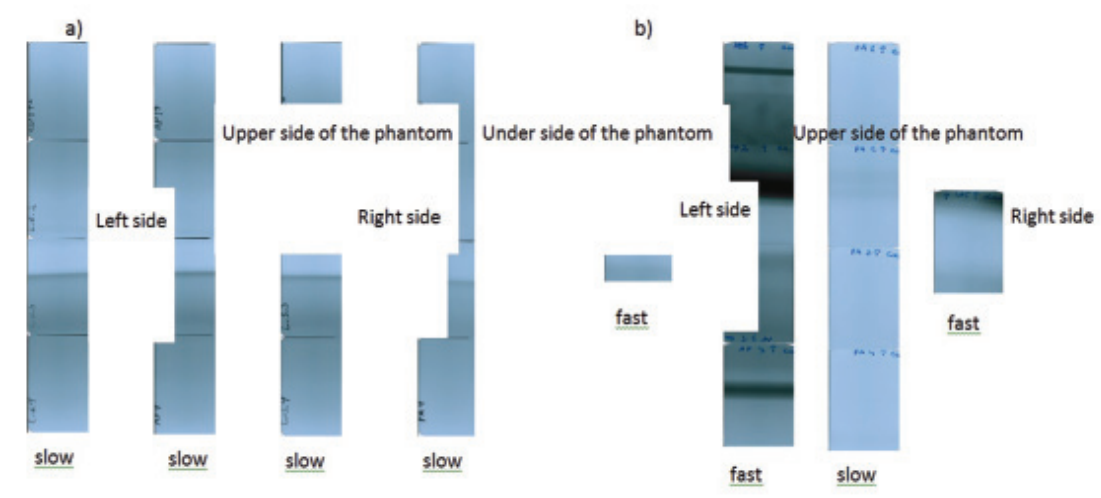

Figure 2. Scanned Personal Monitoring films: a) CT unit A: only slow emulsion is presented due to exposure during the warm-up procedure with over the air kerma range of the fast emulsion, b) CT unit C 


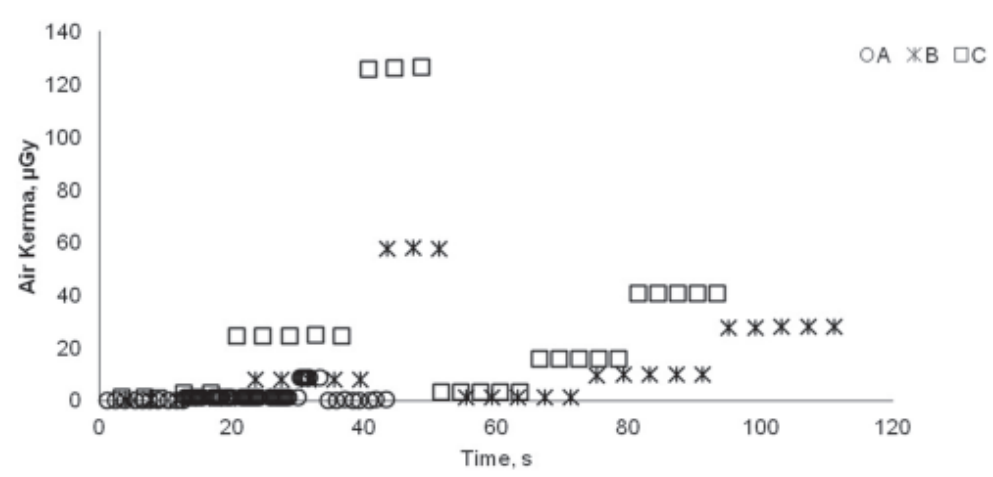

Figure 3. Measured air kerma values during the warm-up process for $\mathrm{CT}$ units $\mathrm{A}, \mathrm{B}$, and $\mathrm{C}$

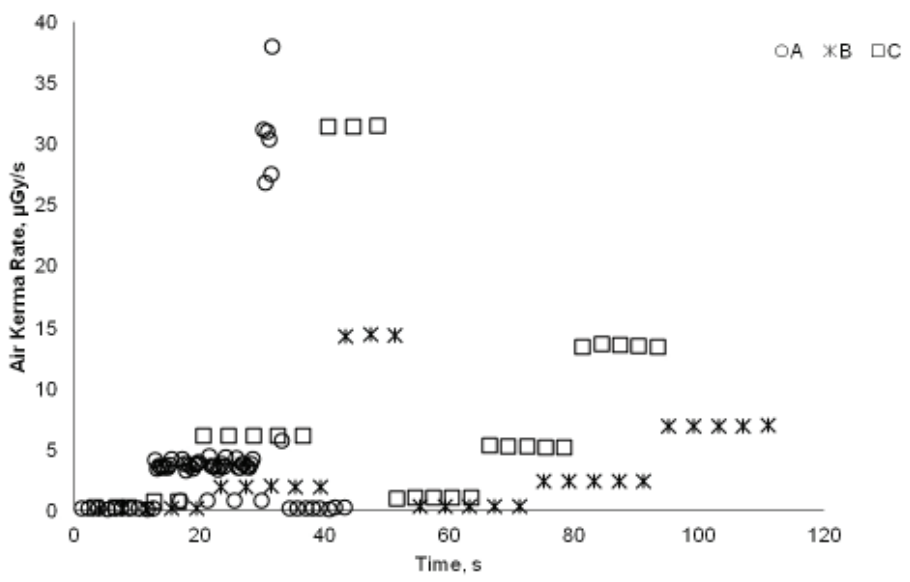

Figure 4. Measured air kerma rate values during the warm-up process for $\mathrm{CT}$ units $\mathrm{A}, \mathrm{B}$, and $\mathrm{C}$

$\mu \mathrm{Gy}$ and $43.27 \mathrm{~s}(\mathrm{~A}), 409 \mu \mathrm{Gy}$, and $111.12 \mathrm{~s}(\mathrm{~B})$ and $807 \mu \mathrm{Gy}$ and $93.40 \mathrm{~s}(\mathrm{C})$. For one of the CT scanners (A), the air kerma rate value during the procedure reached up to $38 \mu \mathrm{Gy}$ s-1. It was found out that units B and C have similar warmup protocols leading from 4 to 8 times higher air kerma values than unit $\mathrm{A}$.

\section{Discussion}

The most crucial principle in radiation protection is to keep radiation exposure and doses „As Low as Reasonably Achievable" (ALARA). In medicine, the accomplishment of this principle is a combination of many parameters like equipment, medical staff experience and training, and acceptable radiation protection practices.

A practice leading to unnecessary patient exposure was observed in three university hospital departments. The main reason to perform a CT X-ray warming procedure in the presence of a patient already positioned on the CT table is usually a combination of two factors: the patient's medical condition and second, the training of the radiographer that such procedure is performed with closed collimators, providing full patient radiation protection. The discussion between radiographers, service engineers, and medical physicists initiated an additional experimental setting to demonstrate that a missing image on the CT monitor display does not guarantee the absence of patient exposure. Our practice demonstrated that few radiology departments are equipped with dosimetry equipment, and additional alternative methods should be used for assessment and visual demonstration of patient exposure in cases of unexpected exposure. One should always keep in mind how to perform appropriate measurements in order to minimize X-ray exposure. In this particular case, a qualitative experimental set-up was carried out to visualize the results [6]. Both experiments proved the medical physicist expectation, but the quantitative evaluation was decisive to the final termination of unnecessary patient exposure due to the easier understanding of the presented results by the medical staff. Another benefit of this discussion was the confirmation of the 
need for close collaboration between medical physicists and medical specialists. Besides the patient, it is often possible for medical staff to be inside the procedure room, who might think that there is no X-Ray radiation.

Presentations and lectures oriented to the radiographers were organized to raise awareness on the issue and avoid this practice in the future. The results were also presented to the service engineers, and a recommendation for additional collimator quality control test was discussed in cases of such a practice in other CT departments. According to all CT manuals available in the different departments, during the warming-up process of the CT x-ray tube, nobody should be within the gantry and/or in the CT room.

The study's limitation was the dosimetry equipment used, but the purpose was not an exact dose measurement. The $\mathrm{X} 2 \mathrm{R} / \mathrm{F}$ sensor is not designed for CT dose measurements. It is too short for dosimetry, leading to underestimated dose values. However, the authors of the survey wanted immediately to stop the practice of unnecessary exposure using the only available dosimetry equipment in one of the departments. The personal monitoring films were the best tool for a visual demonstration of our results to the radiographers. CR cassettes and traditional $\mathrm{X}$-ray films are widely available in the medical imaging departments, and they also could be used for visual checks for unnecessary exposures of patients and medical staff.

\section{Conclusion}

The widespread use of the CT as an imaging modality has resulted in greater population exposure to ionizing radiation and higher risk for radiation-induced injuries. An example is patient exposures after the emergency warming of the CT X-ray tube, leading to unnecessary exposure and/or the personnel in three different hospitals. Collaboration between a medical physicist, an engineer, and medical specialists and continuous training on radiation protection can raise radiation protection awareness in the radiology departments. Simple radiography equipment could always be used to avoid or demonstrate unnecessary exposure of both patients and medical staff.

\section{References}

1. Pelc NJ, Recent and Future Directions in CT Imaging. Ann Biomed Eng. 2014 Feb; 42(2):260-8.

2. Irving B, Leswick DA, Fladeland D, Lim HJ. Knowing the Enemy: Healthcare Provider Knowledge of CT Radiation Dose and Associated Risks. JMIRS. 2016; 47(3):243-50.

3. Trattner S, Pearson GDN, Chin C, Cody DD, Gupta R, Hess CP et al. Standardization and optimization of CT protocols to achieve low dose. J Am Coll Radiol. 2014 Mar; 11(3):271-8.

4. Sprawls P. The web-based edition of the physical principles of medical imaging. 2nd ed. Madison: Medical Physics Publishing; 1995. Available from http://www.sprawls.org/ppmi2/ XRAYHEAT/.

5. Mihai F, Visan T, Stochioiu A, Bercea S, Scarlat F, Optical sensitivity of the Agfa personal monitoring film to $\mathrm{X}$ and gamma rays under FD-III-D badge filters. J. Optoelectron. Adv. M. 2008; 10(72):3426-9.

6. Toshiba Medical Systems Corporation. Toshiba CT User Manual. 2006-2007. 